\title{
Improving printability of a thermoresponsive hydrogel biomaterial ink by nanoclay addition
}

\author{
Chen $\mathrm{Hu}^{1}$, Lukas Hahn ${ }^{1}$, Mengshi Yang ${ }^{1}$, Alexander Altmann ${ }^{1}$, Philipp Stahlhut ${ }^{2}$, \\ Jürgen Groll $^{2}$, and Robert Luxenhofer ${ }^{1,3, *}$ (i) \\ ${ }^{1}$ Functional Polymer Materials, Chair for Advanced Materials Synthesis, Department of Chemistry and Pharmacy and Bavarian \\ Polymer Institute, Julius-Maximilians-Universität Würzburg, Röntgenring 11, 97070 Würzburg, Germany \\ ${ }^{2}$ Department for Functional Materials in Medicine and Dentistry and Bavarian Polymer Institute, University of Würzburg, \\ Pleicherwall 2, 97070 Würzburg, Germany \\ ${ }^{3}$ Soft Matter Chemistry, Department of Chemistry, Helsinki University, 00014 Helsinki, Finland
}

Received: 18 May 2020

Accepted: 30 August 2020

Published online:

25 September 2020

(C) The Author(s) 2020

\begin{abstract}
As a promising biofabrication technology, extrusion-based bioprinting has gained significant attention in the last decade and major advances have been made in the development of bioinks. However, suitable synthetic and stimuliresponsive bioinks are underrepresented in this context. In this work, we described a hybrid system of nanoclay Laponite XLG and thermoresponsive block copolymer poly(2-methyl-2-oxazoline)-b-poly(2-n-propyl-2-oxazine) (PMeOx-b-PnPrOzi) as a novel biomaterial ink and discussed its critical properties relevant for extrusion-based bioprinting, including viscoelastic properties and printability. The hybrid hydrogel retains the thermogelling properties but is strengthened by the added clay (over $5 \mathrm{kPa}$ of storage modulus and $240 \mathrm{~Pa}$ of yield stress). Importantly, the shear-thinning character is further enhanced, which, in combination with very rapid viscosity recovery $(\sim 1 \mathrm{~s})$ and structure recovery $(\sim 10 \mathrm{~s})$, is highly beneficial for extrusion-based $3 \mathrm{D}$ printing. Accordingly, various 3D patterns could be printed with markedly enhanced resolution and shape fidelity compared to the biomaterial ink without added clay.
\end{abstract}

Handling Editor: Maude Jimenez.

Address correspondence to E-mail: robert.luxenhofer@uni-wuerzburg.de 


\section{GRAPHIC ABSTRACT}
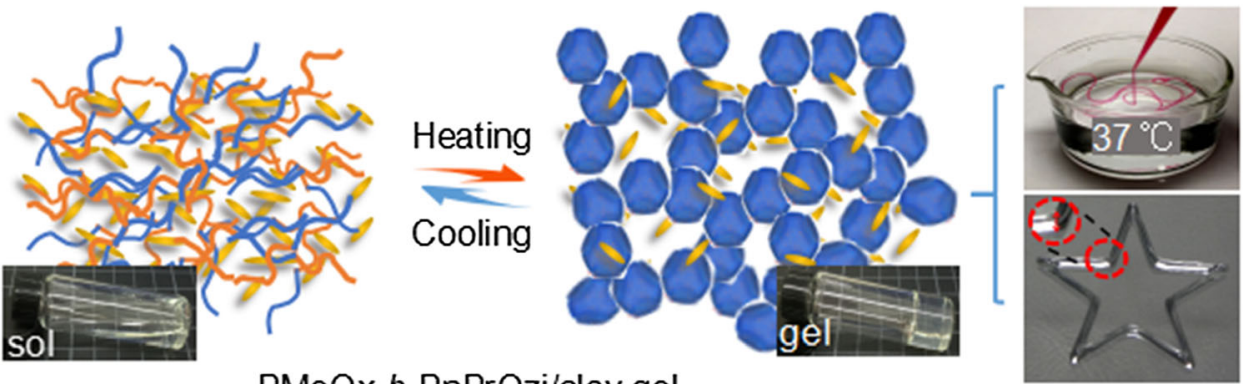

PMeOx-b-PnPrOzi/clay gel

\section{Introduction}

Biofabrication, employed for the engineering of different biological tissues including skin [1], meniscus $[2,3]$, cartilage [3-6], bone [7] and blood vessels [8, 9], attracts more and more attention in the field of tissue engineering [10-12]. As a rapidly growing biofabrication technology, extrusion-based bioprinting has made substantial progress during the last decade as it is compatible with a wide range of build materials and allows on-demand production of 3D scaffolds with precisely controlled architecture [13-16]. However, despite the great progress and remarkable achievements that have been recently made, there are still challenges that hamper its further development, particularly the shortage of high-performance bioinks or biomaterial inks [17] which can achieve high-resolution and high-fidelity printed features without compromising cell viability [14, 18, 19]. Currently, the majority of studies concentrate on a few material classes, specifically naturally derived polymers, such as alginates [20], gelatin [21], collagen [3] and hyaluronic acid [22]. Alternatively, a few synthetic polymers, typically based on polyethylene glycol (PEG) $[23,24]$ and pluronic F127 [9, 25, 26] as well as their mixtures with naturally derived polymers, are utilized [10, 27, 28]. Overall, physically crosslinked synthetic materials are currently underrepresented, even though they can offer excellent control over the rheological properties. In addition, thermoresponsive systems allow even more and triggerable control over this critical parameter. However, it is generally challenging to find an "ideal" combination of printability, shape fidelity, suitable crosslinking method and cytocompatibility. Therefore, the development of novel and versatile bioinks and biomaterial inks, which bring together these diverse prerequisites during and after printing, remains a major research topic within the field of biofabrication.

Poly(2-oxazoline)s (POx)-based biomaterials, synthesized by the living cationic ring-opening polymerization (LCROP), have been discussed as a potential alternative to the more established PEG biomaterial systems, due to their facile synthesis, physicochemical versatility and generally speaking good cyto-/biocompatibility [29-33]. In the last few years, especially chemically crosslinked POx-based hydrogels have been heavily investigated [30, 33-36]. More recently, a few different POx-based block copolymers that undergo thermogelation were described. For example, a diblock copolymer comprising a hydrophilic poly(2-methyl-2-oxazoline) (PMeOx) block and a thermoresponsive poly(2-npropyl-2-oxazine) (PnPrOzi) block was described and used for bioprinting [37]. Alternatively, two different thermoresponsive hydrogels formed by POx ABAtype triblock copolymers were described. One features hydrophilic PMeOx A-blocks and a hydrophobic poly(2-iso-butyl-2-oxazoline) B-block; the other comprises thermoresponsive outer A-blocks of poly(2-n-propyl-2-oxazoline) and a more hydrophilic poly(2-ethyl-2-oxazoline) inner B-block, respectively $[38,39]$. However, it is challenging to print real 3-dimensional constructs at this point, even at a relatively high polymer concentration. This is due to 
suboptimal rheological properties, in particular a low yield point, which allows multilayers to fuse more easily and results in poor shape fidelity. Moreover, high polymer concentrations are considered problematic for cell-laden printing, as it might induce clogging of the nozzle tip and lead to more restrictive environments for the cells (macromolecular crowding) [40-42]. This in turn can negatively affect protein expression, cell viability and proliferation as well as cell migration.

Clay minerals are a group within the phyllosilicates and present in the form of disk-shaped platelets. They are cytocompatible and commonly used as additives to improve mechanical strength and biological effects of soft composites [27, 43-48]. In addition, it can improve printability, as recently pioneered by the groups of Gelinsky [49] and Huang [50], which is of particular interest for the present purpose. Herein, we report a hybrid system of Laponite XLG and a recently presented synthetic thermoresponsive block copolymer PMeOx- $b$ PnPrOzi [37] as a potential biomaterial ink formulation and discuss its critical properties relevant for extrusion-based bioprinting including viscoelastic properties and printability. Notably, the thermoresponsibility is not critically affected while its rheological properties are markedly improved in terms of storage modulus, yield stress and shear thinning. These effects are highly desirable for extrusion-based 3D (bio)printing technology and result in a significantly improved printability. Apart from expanding the options available to researchers for 3D bioprinting, this novel thermoresponsive hydrogel composite may also provide a new and promising material platform for other research fields, such as controlled drug delivery and release [51], versatile support bath material for fluid extrusion printing [52], sacrificial material templates in fabrication of microfluidic devices [53] and thermoresponsive self-protection [54].

\section{Materials and methods}

\section{Materials}

All substances and reagents were obtained from Sigma-Aldrich (Steinheim, Germany) and Acros (Geel, Belgium). Zinc acetate dihydrate, butyronitrile, 3-amino-1-propanol, 1-Boc-piperazine (Boc-Pip) and potassium carbonate were used as received. Reagents used for polymerization, specifically methyl trifluoromethylsulfonate (MeOTf), 2-methyl-2-oxazoline $(\mathrm{MeOx})$, benzonitrile $(\mathrm{PhCN})$, were dried by refluxing over $\mathrm{CaH}_{2}$ under dry argon atmosphere and subsequent distillation before use. The monomer 2- $n$ propyl-2-oxazine (nPrOzi) was synthesized by an adapted standard procedure [55], distilled over $\mathrm{CaH}_{2}$ and stored under dry argon. Laponite XLG ([ $\mathrm{Mg}_{5.34-}$ $\left.\mathrm{Li}_{0.66} \mathrm{Si}_{8} \mathrm{O}_{20}(\mathrm{OH})_{4}\right] \mathrm{Na}_{0.66}$ ) was purchased from BYKChemical $\mathrm{GmbH}$ (Wesel, Germany) and used as received. Deionized water was used throughout the experiments.

\section{Synthesis of PMeOx-b-PnPrOzi diblock copolymers}

The PMeOx- $b$-PnPrOzi diblock copolymer was synthesized using MeOTf to initiate a cationic ringopening polymerization of $\mathrm{MeOx}$ and $\mathrm{nPrOzi}$, as previously described (Figure S1) [37, 56]. Briefly, under dry and inert conditions, $50 \mu \mathrm{L}(0.47 \mathrm{mmol}$, 1 eq.) MeOTf and $3.99 \mathrm{~g}$ (46.88 mmol, 100 eq.) MeOx were added to $9.43 \mathrm{~mL}$ of dry $\mathrm{PhCN}$ in a dried flask and polymerized at $120{ }^{\circ} \mathrm{C}$ for $4 \mathrm{~h}$. Full monomer conversion was verified by ${ }^{1} \mathrm{H}$ NMR before addition of the monomer for the second block. After the reaction mixture cooled to ambient temperature, $5.99 \mathrm{~g} \mathrm{nPrOzi}$ (47.14 mmol, 100 eq.) dissolved in $11.67 \mathrm{~mL}$ of dry PhCN was added and stirred at $140{ }^{\circ} \mathrm{C}$ overnight. Subsequently, the mixture was cooled again to room temperature, and $0.26 \mathrm{~g}$ (1.4 mmol, 3 eq.) Boc-Pip was added. The reaction mixture was stirred overnight at $40{ }^{\circ} \mathrm{C}$. After cooling to room temperature, $60 \mathrm{mg}$ potassium carbonate ( $0.43 \mathrm{mmol}, 1$ eq.) was added and the mixture was again stirred for $5 \mathrm{~h}$. Thereafter, the solvent was removed at reduced pressure, the residual dissolved in deionized water and dialyzed against deionized water using a dialysis membrane (molecular weight cutoff $(\mathrm{MWCO})=10 \mathrm{kDa})$ for 2 days. The product was obtained as an off-white powder after lyophilization (9.06 g, 74\% yield).

The chemical structure of PMeOx- $b$-PnPrOzi was confirmed by ${ }^{1} \mathrm{H}$ NMR on a Fourier 300 (300.12 MHz) Bruker BioSpin (Rheinstetten, Germany) system at $298 \mathrm{~K}$ with $\mathrm{CDCl}_{3}$ as solvent. Gel permeation chromatography (GPC) was performed on an Agilent 1260 Infinity, System Polymer Standards Service (Mainz, Germany) with hexafluoroisopropanol 
(HFIP) containing $3 \mathrm{~g} \mathrm{~L}^{-1}$ potassium trifluoroacetate (KTFA) as eluent, precolumn: $50 \mathrm{~mm} \times 8 \mathrm{~mm}$ PSS PFG linear M; 2 columns: $300 \mathrm{~mm} \times 8 \mathrm{~mm}$ PSS PFG linear $\mathrm{M}$ (particle size $7 \mu \mathrm{m}$; pore size $0.1-1000 \mathrm{kDa}$ ). The columns were kept at $40^{\circ} \mathrm{C}$, and flow rate was

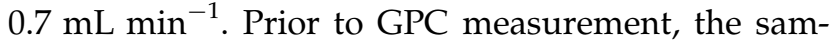
ple was filtered through a $0.2-\mu \mathrm{m}$ Teflon filter (Thermo Scientific) to remove particles, if any.

\section{Preparation of PMeOx-b-PnPrOzi/clay Hybrid Hydrogels}

The nanocomposite PMeOx- $b$-PnPrOzi/clay hybrid hydrogels were prepared by thermogelation of an aqueous dispersion consisting of Laponite XLG and PMeOx-b-PnPrOzi copolymers. Specifically, $2 \mathrm{wt} \%$ Laponite XLG aqueous sol was prepared by dispersing the appropriate amount of dry Laponite XLG powder in deionized water with continuous stirring to ensure the thorough hydration of Laponite powder, until a homogeneous and transparent clay sol was obtained (at least $24 \mathrm{~h}$ ). The sol was stored in sealed containers to prevent evaporation and shaken before use. Subsequently, the dry PMeOx-b-PnPrOzi powder was added to the $2 \mathrm{wt} \%$ clay sol to reach the desired polymer final concentration $(15,16,18,20$ and $30 \mathrm{wt} \%$ ) and stored at $7{ }^{\circ} \mathrm{C}$ overnight to allow complete dissolution (Table S1, Supporting Information). Accordingly, the final clay concentration also changed with different polymer concentrations. For example, at $15 \mathrm{wt} \%$ polymer, the clay concentration was $1.7 \mathrm{wt} \%$, while at $30 \mathrm{wt} \%$ polymer, the clay concentration was $1.4 \mathrm{wt} \%$. Afterwards, the mixture sol can undergo a thermogelation at room temperature rapidly and form the stable $\mathrm{PMeOx}-b$-PnPrO$\mathrm{zi} /$ clay hybrid hydrogels. For reference, a $20 \mathrm{wt} \%$ PMeOx- $b$-PnPrOzi hydrogel without clay was also prepared accordingly. Unless otherwise noted, the hybrid hydrogels used throughout this study comprised PMeOx-b-PnPrOzi at a concentration of $20 \mathrm{wt} \%$ and a final clay concentration of $1.6 \mathrm{wt} \%$. All samples were stored at $7{ }^{\circ} \mathrm{C}$ until used.

\section{Characterization of hydrogels}

Cryogenic scanning electron microscopy (Cryo-SEM) on hydrogel samples was performed using a Zeiss Crossbeam 340 field emission SEM (Oberkochen, Germany), where the as-prepared samples were flash-frozen $(2 \times 1 \mathrm{~mm}$ pellet $)$ in nitrogen slush at
- $210{ }^{\circ} \mathrm{C}$. Samples were freeze fractured and freeze etched at $-85^{\circ} \mathrm{C}$ for $15 \mathrm{~min}$ and sputtered subsequently with platinum $(3 \mathrm{~nm})$ before observation (at $-140{ }^{\circ} \mathrm{C}$ ). The pure clay was imaged using standard scanning electron microscopy at room temperature. The SEM images were obtained at an acceleration voltage of $2.00 \mathrm{kV}$ for pure clay and $8.00 \mathrm{kV}$ for all hydrogel samples. X-ray diffraction (XRD) was analyzed with a Bruker D8 Discover powder diffractometer (Karlsruhe, Germany) in the range of $5^{\circ}-60^{\circ}$ (20). A UV-vis-NIR Cary 5000 from Agilent Technologies (Santa Clara, USA) was used to measure the optical transparency of the as-prepared hydrogels at $37^{\circ} \mathrm{C}$. Thermogravimetric analysis (TGA) of the hydrogels was performed on a thermal gravimetric analyzer from Netzsch (Selb, Germany) under nitrogen flow. The hydrogels were heated from 30 to $900{ }^{\circ} \mathrm{C}$ with a heating rate of $10 \mathrm{~K} \mathrm{~min}^{-1}$. Differential scanning calorimetry (DSC) measurements were performed in aluminum crucibles on a DSC 204 F1 Phoenix system from Netzsch (Selb, Germany). The samples were heated to $240{ }^{\circ} \mathrm{C}$ and subsequently followed by two additional times of heated/cooled from - 50 to $200{ }^{\circ} \mathrm{C}$, where the heating and cooling rate was $10 \mathrm{~K} \mathrm{~min}^{-1}$ in a constant $\mathrm{N}_{2}$-atmosphere.

\section{Rheological measurement}

Rheological measurements were performed using the MCR 301 rheometer from Anton Paar (Ostfildern, Germany) employing a 25-mm-diameter parallel plate geometry. A Peltier system was employed for temperature control. First, at a constant angular frequency and strain of $10 \mathrm{rad} \mathrm{s}^{-1}$ and $0.5 \%$, respectively, temperature sweeps from 5 to $45{ }^{\circ} \mathrm{C}$ were carried out and immediately followed by a cooling process from 45 to $5{ }^{\circ} \mathrm{C}$. The heating and cooling rate was $0.05^{\circ} \mathrm{C} \mathrm{s}^{-1}$. In preliminary work, we also tested slower heating/cooling rates, but not difference was observed. Then, six kinds of rheological experiments were performed at $37^{\circ} \mathrm{C}$. (1) Strain sweeps with oscillation strain amplitudes of $0.01-500 \%$ at a constant angular frequency of $10 \mathrm{rad} \mathrm{s}^{-1}$, from which the linear viscoelastic (LVE) range of the samples can be determined. (2) Frequency sweeps in angular frequency range of $0.1-100 \mathrm{rad} \mathrm{s}^{-1}$ at a constant strain of $0.1 \%$. (3) Time sweeps with alternating oscillation strain of $0.1 \%$ and $100 \%$ at a constant angular frequency of $10 \mathrm{rad} \mathrm{s}^{-1}$. (4) Steady-state shear flow from 0.01 to $1000 \mathrm{~s}^{-1}$ of shear rate. (5) Time sweeps of 
shear flow with alternating shear rate of $0.1 \mathrm{~s}^{-1}$ and $100 \mathrm{~s}^{-1}$. (6) Hysteresis loop tests in shear rate range of 0 to $500 \mathrm{~s}^{-1}$. An aqueous solvent trap was utilized in all experiments to mitigate drying effects.

\section{$3 \mathrm{D}$ printing}

Printing experiments were performed using the extrusion-based 3D bioprinter Inkredible (CellInk, Gothenburg, Sweden) equipped with a pneumaticdriven print head (syringe dispenser, DD-135N) and a 0.20-mm-inner diameter precision needle from Nordson EFD (Oberhaching, Germany). The extrusion pressure was controlled and varied from 110 to $220 \mathrm{kPa}$ according to different user-defined printing structures which are programmed by G-code. The hydrogels were loaded into a printing cartridge in liquid form immediately after taking out from the $7{ }^{\circ} \mathrm{C}$ refrigerator, mounted in the $3 \mathrm{D}$ printer and driven pneumatically through the nozzle to print the hydrogel onto polystyrene plates at room temperature. The printing speed was fixed at $600 \mathrm{~mm} \mathrm{~min}^{-1}$ in all printing experiments. The printed constructs were imaged using a stereomicroscope Carl Zeiss Discovery V.20 (Jena, Germany) to visually characterize the shape fidelity post-fabrication.

\section{Results and discussion}

\section{Synthesis and characterization of PMeOx-b- PnPrOzi diblock copolymers}

The diblock copolymer PMeOx- $b$-PnPrOzi was synthesized by LCROP from the monomers $\mathrm{MeOx}$ and nPrOzi according to our previous reported procedure and characterized (Fig. 1a and Figure S1, Supporting Information) [37, 56]. From the signals in the ${ }^{1} \mathrm{H}$ NMR spectrum with chemical shifts at $2.14 \mathrm{ppm}\left(\mathrm{CH}_{3}\right.$ of PMeOx) and 0.95 ppm $\left(\mathrm{CH}_{3}\right.$ of PnPrOzi), the relative block lengths and diblock copolymer composition were determined to be $\mathrm{PMeOx}_{116}-b$-PnPrOzi ${ }_{125}$ with a number-average molar mass $\left(\mathrm{M}_{\mathrm{n}}\right)$ of about $26 \mathrm{~kg} \mathrm{~mol}^{-1}$ and a dispersity of $D=1.28$ (obtained form GPC), indicating that the desired polymer was obtained with reasonable control and definition (Fig. 1b, c). The analytical data are listed in Table 1.

\section{Preparation and characterization of $\mathrm{PMeOx}-$ b-PnPrOzi/clay hydrogels}

The PMeOx-b-PnPrOzi/clay hydrogels were prepared by dissolving the synthesized PMeOx- $b$ PnPrOzi block copolymer in a $2 \mathrm{wt} \%$ aqueous clay sol at $7{ }^{\circ} \mathrm{C}$ overnight and then incubation at room temperature to trigger the thermogelation (Fig. 2a). According to our previous study, when the block copolymer solution is above its critical gelation concentration (20 wt\%), sol-gel transition will occur at a critical gelation temperature $\left(T_{\text {gel }}\right)[37,38]$. Similarly, the prepared PMeOx-b-PnPrOzi/clay sols showed reversible thermogelation (Fig. 2b; Video S1, Supporting Information). To illustrate the fast in situ thermogelation and injectability of $\mathrm{PMeOx}-b$-PnPrO$\mathrm{zi} /$ clay hydrogel, we pipetted it into a water reservoir $\left(37^{\circ} \mathrm{C}\right)$ and found that clear hydrogel strands formed immediately after injecting into the water (Fig. 2c; Video S2, Supporting Information). However, after a few minutes we observed that the strands dissolved gradually due to dilution.

In the absence of clay, the block copolymer selfassembles into spherical vesicles of several hundred nm of size. Above a critical concentration and temperature, a sponge-like network leading the macroscopic physical gelation was observed [37, 57]. We hypothesize that the clay nanoparticles interact with these vesicles, probably adsorbing to their surface via hydrogen bonding. XRD experiments confirmed that the block copolymer interacts strongly with the clay platelets as shown by the disappearance of the aggregation peak of clay (Figure S2, Supporting Information). Most interestingly, the incorporation of clay into the polymer matrix did not reduce the transparency of gels, which indicated that the clay platelets were uniformly dispersed in the polymer matrix (Figure S3, Supporting Information). When decreasing the temperature, the sol-gel transition is reversed and the PMeOx-b-PnPrOzi/clay hydrogel liquefies again.

The composition and thermal properties of the freeze-dried hydrogels were investigated by TGA and DSC (Figure S4, Supporting Information). As expected, the clay showed little thermal decomposition with only a slight weight loss due to moisture (92 wt\% remained for clay at $900{ }^{\circ} \mathrm{C}$ ). For PMeOx- $b$ PnPrOzi and the PMeOx-b-PnPrOzi/clay nanocomposite, pronounced weight loss was found between 300 and $700{ }^{\circ} \mathrm{C}$ corresponding to the decomposition 
(a)

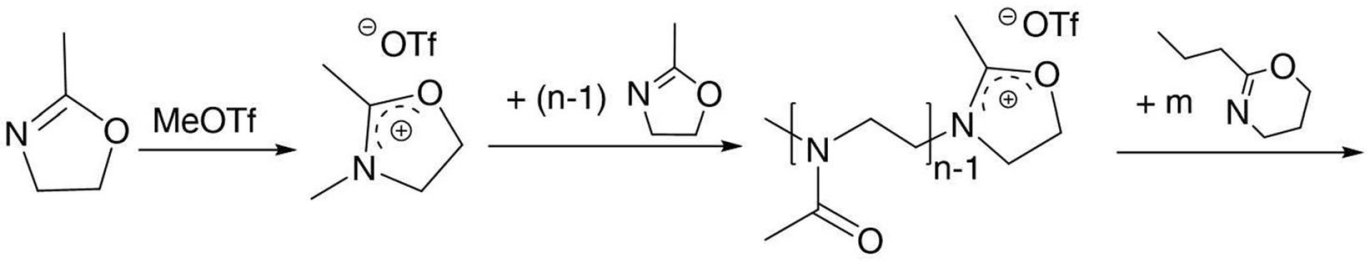

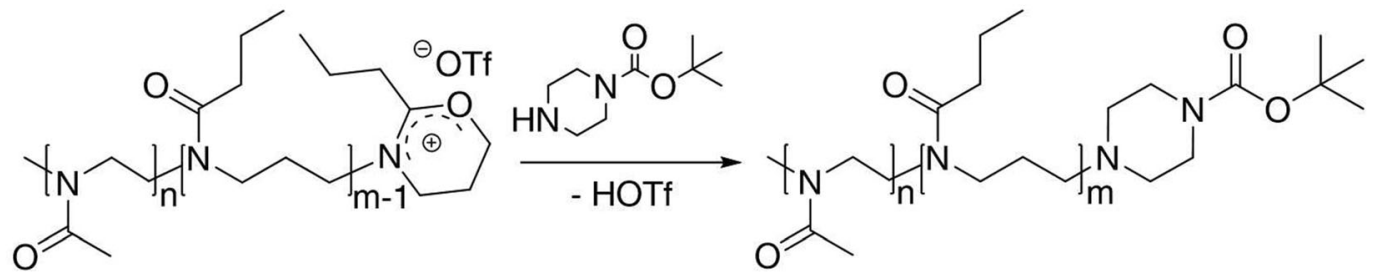

(b)

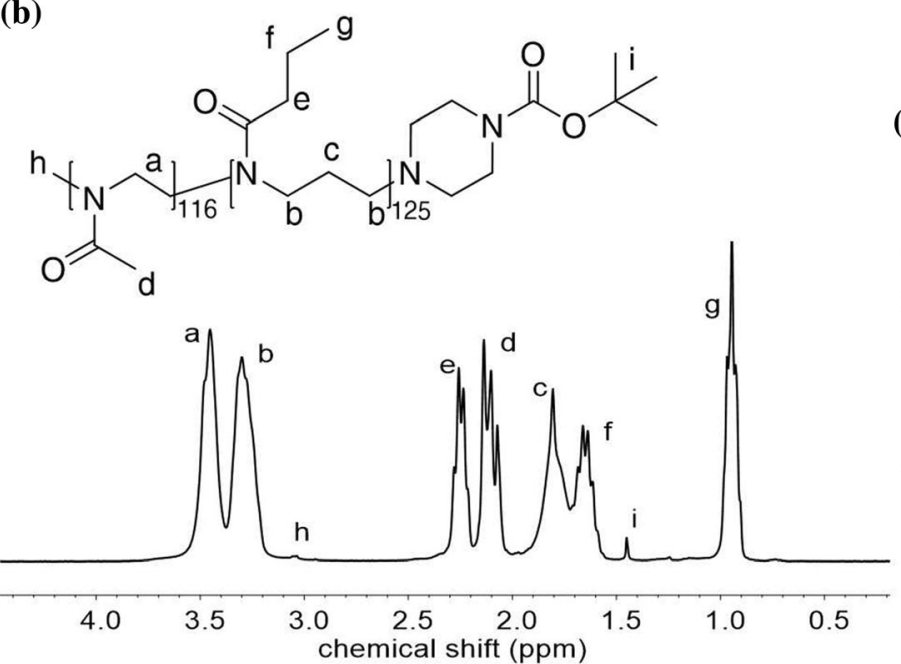

(c)

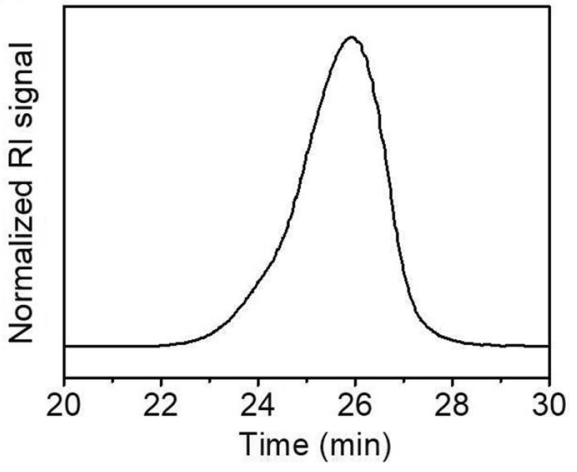

Figure 1 a Schematic presentation of the synthesis of diblock copolymer PMeOx- $b$-PnPrOzi. b Chemical structure of the synthesized diblock copolymer PMeOx- $b$-PnPrOzi and the

Table 1 Polymer composition, molar masses $\left(\mathrm{kg} \mathrm{mol}^{-1}\right)$ and dispersity of the synthesized PMeOx- $b$-PnPrOzi diblock copolymer obtained via ${ }^{1} \mathrm{H}$ NMR and GPC with HFIP as eluent

\begin{tabular}{lllll}
\hline Polymer composition, $\exp ^{\mathrm{a}}$ & $\mathrm{M}_{\mathrm{n}}^{\mathrm{a}}$ & $\mathrm{M}_{\mathrm{n}}{ }^{\mathrm{b}}$ & $\mathrm{M}_{\mathrm{w}}{ }^{\mathrm{b}}$ & $D^{\mathrm{b}}$
\end{tabular}

$\begin{array}{lllll}\mathrm{Me}_{-} \mathrm{PMeOx}_{116}-b-\mathrm{P} n \mathrm{PrOzi}_{125} \text {-Boc-Pip } & 26 & 7.7 & 9.9 & 1.28\end{array}$

${ }^{a}$ Determined by end-group analysis $\left({ }^{1} \mathrm{H}\right.$ NMR spectroscopy in $\mathrm{CDCl}_{3}, 300 \mathrm{MHz}, 298 \mathrm{~K}$ )

${ }^{\mathrm{b}}$ Determined from GPC in HFIP with KTFA $\left(3 \mathrm{~g} \mathrm{~L}^{-1}\right)$ at $313 \mathrm{~K}$ and calibrated against poly(ethylene glycol) standards

of the PMeOx- $b$-PnPrOzi component in the hydrogels. The degradation onset was $300{ }^{\circ} \mathrm{C}$ and $285^{\circ} \mathrm{C}$, respectively. Even though this effect is minor and irrelevant for the application in a hydrogel, it corresponding ${ }^{1} \mathrm{H}$ NMR spectrum. c GPC elution profile of the synthesized diblock copolymer.

indicates that components in the clay lead to an earlier thermal degradation of PMeOx- $b$-PnPrOzi. The difference in residual mass at $900{ }^{\circ} \mathrm{C}$ reflects the composition of the PMeOx- $b$-PnPrOzi/clay nanocomposite. Compared with the pristine $\mathrm{PMeOx}-$ $b$-PnPrOzi, an extra residual of 8.5 wt $\%$ for PMeOx- $b$ $\mathrm{PnPrOzi} /$ clay was observed and attributed to the added clay, which is comparable to the theoretical composition of clay $(8 \mathrm{wt} \%)$ in $\mathrm{PMeOx}-b-\mathrm{PnPrOzi} /-$ clay system. In this work, the glass transition temperature $\left(T_{\mathrm{g}}\right)$ is defined as the midpoint of the step in the heating curve. For the block copolymer, two glass transition points were found because of the (micro)phase separation of the PMeOx block and the PnPrOzi block in bulk. In the case of PMeOx- $b$ PnPrOzi/clay, we observed a very minor decrease in 
Figure 2 a Schematic diagram for preparation of PMeOx- $b$-PnPrOzi/clay hydrogel. b Photographs indicating the thermal reversible sol-gel transition of $\mathrm{PMeOx}-b$-PnPrOzi/clay hydrogel. c Photograph demonstrating the injectable and in situ thermal gelation properties of $\mathrm{PMeOx}$ $b$-PnPrOzi/clay hydrogel (dyed red by carmine). (a)

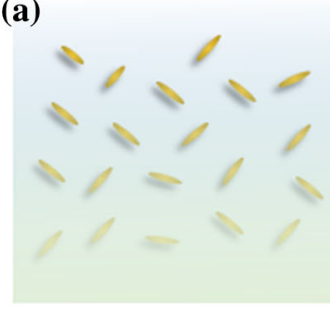

aqueous clay sol

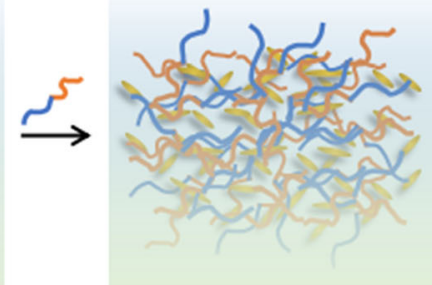

PMeOx-b-PnPrOzi/clay sol

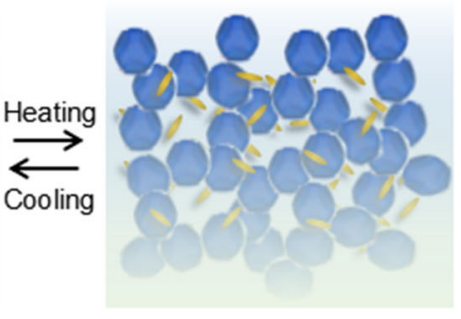

PMeOx-b-PnPrOzi/clay gel

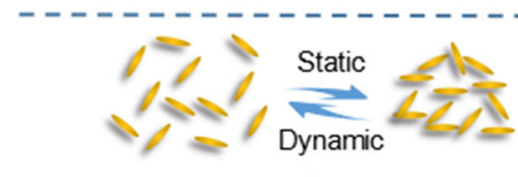

(b)

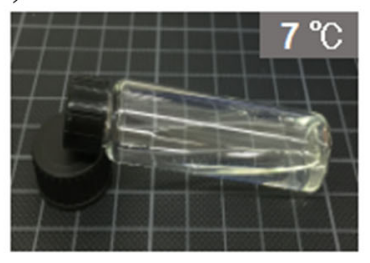

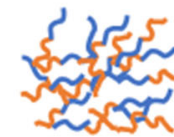

PMeOx-b-PnPrOzi

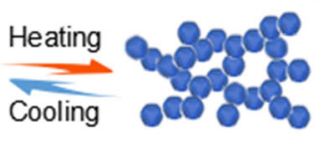

(c)

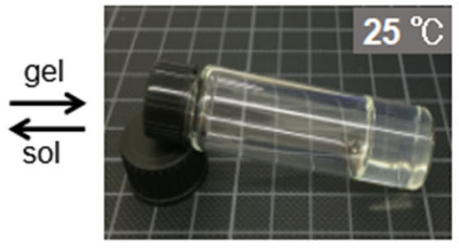

the first $T_{\mathrm{g}}$ of the PnPrOzi block, while the second $T_{\mathrm{g}}$ of the PMeOx block appears to be attenuated more profoundly. This may indicate that the added clay preferentially interacts with PMeOx. Very recently, a report by Le Coeur et al. investigated the interaction of PMeOx and Laponite RD. The authors found a stronger interaction of $\mathrm{PMeOx}$ with the clay compared to poly(ethylene glycol) and clay [58].

\section{Rheological properties of PMeOx- $b$ - PnPrOzi/clay hydrogels}

Clay minerals such as Laponite are well-known rheology modifiers and recently introduced specifically for the improvement in 3D printability of bioinks $[49,50]$. Previously, pluronic F127 has been combined with Laponite RDS and investigated rheologically. While at $1 \mathrm{wt} \%$ of Laponite only minor effects were observed, the gelation temperature increased significantly and the storage modulus decreased when 2 and $3 \mathrm{wt} \%$ Laponite RDS were added [59]. Even though the authors did not investigate this, one would have to assume that such effect would be detrimental to the printability of pluronic F127. Apart from the very recent report by Le Coeur [58], the interaction of POx and clay has not been studied in considerable detail. Important to note, Le Coeur found a stronger interaction of $\mathrm{PMeOx}$ with clay compared to the interaction of poly(ethylene glycol) and clay. Therefore, it is of great interest to understand how clay affects the temperature-dependent rheological properties of PMeOx- $b$-PnPrOzi. Accordingly, we studied the rheological and viscoelastic behavior of PMeOx- $b$-PnPrOzi/clay hydrogels in detail, to assess the effects under dynamic printing conditions.

Initially, the $20 \mathrm{wt} \% \quad \mathrm{PMeOx}-b$-PnPrOzi/clay hydrogel was chosen as a representative concentration and the $20 \mathrm{wt} \%$ pristine PMeOx-b-PnPrOzi hydrogel as control. First, to characterize the thermogelling behavior, a temperature sweep was performed by recording storage modulus $\left(G^{\prime}\right)$ and loss modulus $\left(G^{\prime \prime}\right)$ under dynamic oscillation conditions (Fig. 3a), in which the intersection of $G^{\prime}=G^{\prime \prime}$ defines the gel point and gelation temperature $\left(T_{\text {gel }}\right)$. At low temperature, $G^{\prime \prime}>G^{\prime}$ indicates a sol state. As the temperature rises, both moduli increase, first moderately and then rapidly close to the $T_{\text {gel }}$ (15 and $17^{\circ} \mathrm{C}$, respectively), before reaching plateau values at above $21-25^{\circ} \mathrm{C}$, which is consistent with the observations in the test tube inversion experiments. The stable plateau extends well beyond $37^{\circ} \mathrm{C}$ (physiological temperature). In contrast to the pristine PMeOx- $b$-PnPrOzi hydrogel of $T_{\text {gel }}$ around $17^{\circ} \mathrm{C}$ and $G_{\max }^{\prime} 3.3 \pm 0.2 \mathrm{kPa}(n=3), \mathrm{PMeOx}-b$-PnPrOzi/clay hydrogel exhibits a slightly lower $T_{\text {gel }}$ around $15^{\circ} \mathrm{C}$ and a well enhanced $G^{\prime}{ }_{\text {max }} 5.3 \pm 0.3 \mathrm{kPa}(n=3)$ due to the introducing of clay.

A heating-cooling cycle for the PMeOx- $b$-PnPrOzi/clay hydrogel (Fig. $3 b$ ) revealed a small hysteresis 
Figure 3 a Temperaturedependent rheological properties of $\mathrm{PMeOx}-b$ PnPrOzi and $\mathrm{PMeOx}-b$ PnPrOzi/clay hydrogels. b A cyclic temperature sweep of $\mathrm{PMeOx}-b$-PnPrOzi/clay hydrogel under a heating/cooling rate $0.05{ }^{\circ} \mathrm{C} \mathrm{s}^{-1}$. c Temperaturedependent rheological properties of $\mathrm{PMeOx}-b$ PnPrOzi/clay hydrogels with varying compositions.

d Composition-dependent thermogelation properties of $\mathrm{PMeOx}-b$-PnPrOzi/clay hydrogels.
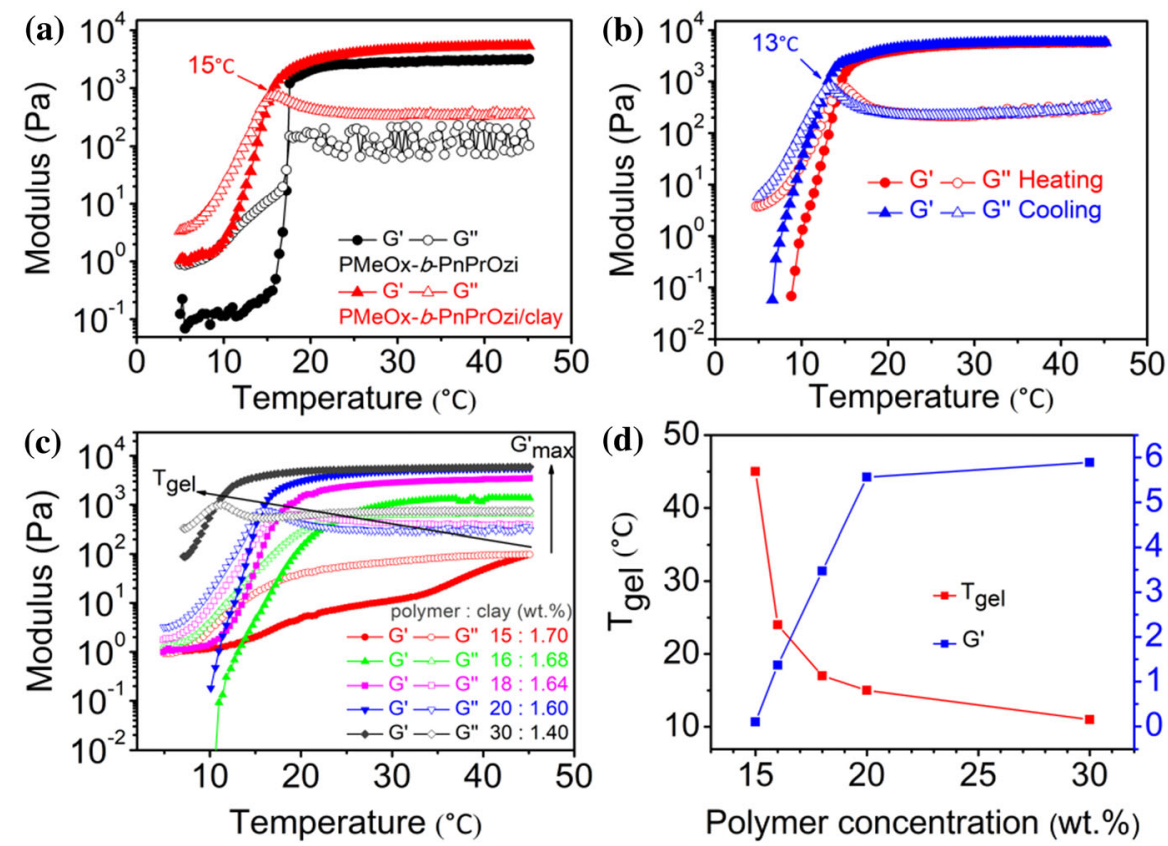

of only $2 \mathrm{~K}$ for the $T_{\text {gel }}$. Above $T_{\text {gel, }}$ the moduli of heating and cooling cycles overlapped very well. The small hysteresis of the modulus below $T_{\text {gel }}$ attributes to the formation of extra physical interactions between the copolymers and clay in nanocomposite system during the sol-gel transition. Next, we investigated the thermogelling properties of PMeOx$b$-PnPrOzi/clay hydrogels at different block copolymer/clay concentrations (Fig. 3c). The $G^{\prime}{ }_{\max }$ increases with increasing polymer concentration and stabilizes at around 5-6 kPa eventually, while the $T_{\text {gel }}$ decreases with increasing polymer concentration (Fig. 3d). Interesting to note, the critical thermal gelation concentration of PMeOx- $b$-PnPrOzi/clay was decreased to $15 \mathrm{wt} \%$ (plus $1.7 \mathrm{wt} \%$ clay) from $20 \mathrm{wt} \%$ for the pristine PMeOx-b-PnPrOzi, which is beneficial as the clay additive is much cheaper and commercially available compared to the block copolymer. Gao et al. [60] proposed the loss factor $\left(G^{\prime \prime} / G^{\prime}=\tan \delta\right)$ to assess the printability. They used gelatin-alginate composite as model hydrogels and defined a range between 0.25 and 0.45 as excellent compromise between extrusion uniformity and structural integrity. Accordingly, the $\tan \delta$ values of hydrogels with varying compositions in this study were extracted (Table S2, Supporting Information). It is obvious that $15 \mathrm{wt} \%$ (with $1.7 \mathrm{wt} \%$ clay) PMeOx-b$\mathrm{PnPrOzi} /$ clay hydrogel is more viscous in character $\left(\tan \delta \approx 1 ; G^{\prime}<0.1 \mathrm{kPa}\right)$, though it did form gel
$\left(G^{\prime} \geq G^{\prime \prime}\right)$. Nevertheless, all the other compositions formed gels with a more elastic character $(\tan \delta<$ $0.5)$, especially the $20 \mathrm{wt} \% \mathrm{PMeOx}-b$-PnPrOzi/clay hydrogel with $1.6 \mathrm{wt} \%$ clay additives $(\tan \delta \approx 0.05$; $G^{\prime} \approx 5.5 \mathrm{kPa}$ ). In comparison, the $\tan \delta$ of the pristine $20 \mathrm{wt} \% \mathrm{PMeOx}-b$-PnPrOzi without clay is somewhat lower $(\approx 0.04)$. Clearly, these values lie significantly outside the range suggested by Gao and Gillispie.

The dynamic strain sweeps were conducted to determine the LVE range, where the plateau of $G^{\prime}$ and $G^{\prime \prime}$ is commonly referred to the LVE region. It is obvious that the apparent LVE range for PMeOx- $b$ PnPrOzi/clay hydrogel (shaded pink area) extends further than that of the pristine PMeOx-b-PnPrOzi hydrogel (shaded gray area) (Fig. 4a), suggesting that the $\mathrm{PMeOx}-b$-PnPrOzi/clay hydrogel is more elastic and stable to oscillating strain. The onset of the decrease in $G^{\prime}$ and increase in $G^{\prime \prime}$ indicates damage to the hydrogels inner structure. Therefore, a strain value of $0.1 \%$ (within the LVE range) was chosen for the subsequent dynamic frequency sweep experiments (Fig. $4 b$ ). Here, $G^{\prime}$ was relatively stable and higher than $G^{\prime \prime}$ in the whole investigated frequency range (0.1-100 $\left.\mathrm{rad} \mathrm{s}^{-1}\right)$, demonstrating that both PMeOx- $b$-PnPrOzi and PMeOx- $b$-PnPrOzi/clay exhibited and maintained the typical gel phase from low to high shear frequency. Again, the storage modulus $G^{\prime}$ of the PMeOx- $b$-PnPrOzi/clay hydrogel is about 2 times higher than $G^{\prime}$ of PMeOx-b-PnPrOzi, 

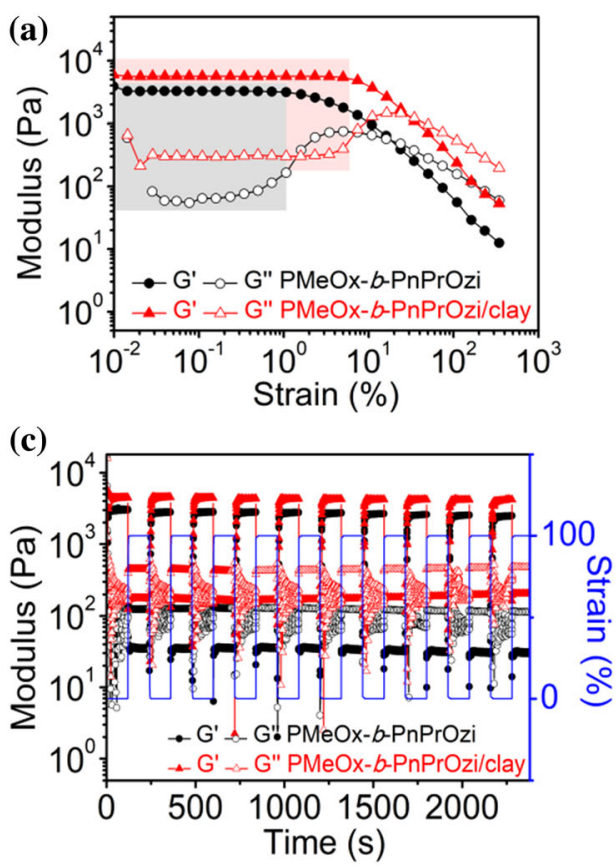

Figure 4 a Amplitude sweeps of hydrogels, where the marked gray and pink areas represent the LVE range of $\mathrm{PMeOx}-b$-PnPrOzi and $\mathrm{PMeOx}-b$-PnPrOzi/clay hydrogels, respectively. b Dependence of $G^{\prime}$ and $G^{\prime \prime}$ on the angular frequency of hydrogels. c Structure recovery testing for hydrogels under

corroborating earlier results and supporting the idea of additional physical interaction, i.e., hydrogen bonds between PMeOx- $b$-PnPrOzi copolymer chains and clay, which contribute to the increase in $G^{\prime}$.

Overall, the rheological analysis shows that the PMeOx- $b$-PnPrOzi/clay hydrogel contains stronger physical crosslinks under dynamic external forces than the pristine PMeOx-b-PnPrOzi hydrogel. Despite these stronger physical crosslinks, the transition between sol and gel states is essentially reversible within $10 \mathrm{~s}$, which was confirmed through the structure recovery testing under alternant strains of $0.1 \%$ and $100 \%$ (Fig. 4c, d). Shear thinning is a very important property of materials for 3D (bio)printing. In the steady-state flow test, it was observed that the viscosity of hydrogels decreased very markedly with the increasing shear rate, indicating a pronounced shear-thinning behavior (Fig. 5a). The flow index $n$ and the consistency index $K$ obtained by fitting the shear rateviscosity rheology plot using the power-law expression show that PMeOx- $b$-PnPrOzi/clay
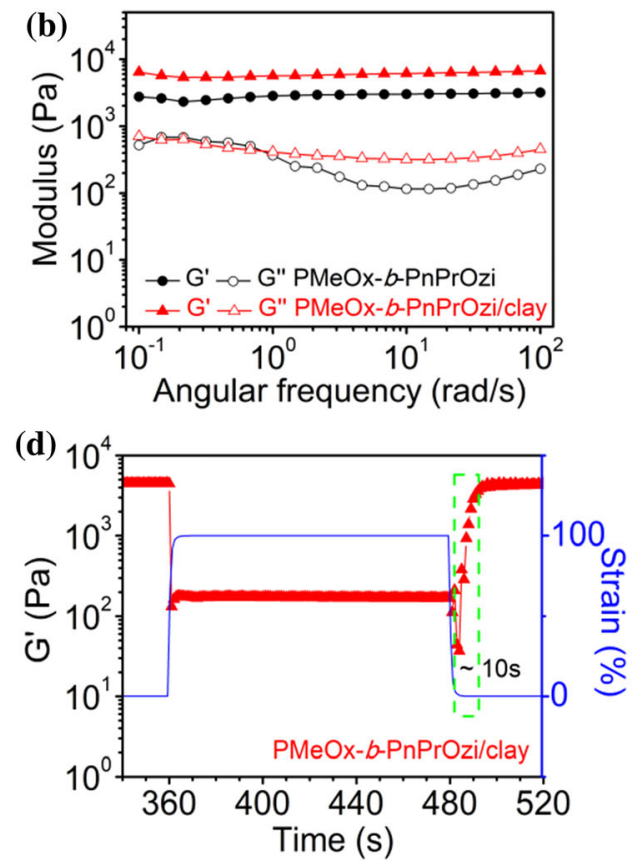

applied alternant strains of $0.1 \%$ and $100 \%$. d Enlarged $G^{\prime}$ curve of PMeOx- $b$-PnPrOzi/clay hydrogel in structure recovery testing results for better visibility of the recovery property. The green dashed frame highlights the time needed for full structure recovery. All experiments were carried out at $37{ }^{\circ} \mathrm{C}$.

hydrogel $(n=0.02 ; K=367.2)$ has an enhanced shear-thinning character, compared to pristine PMeOx- $b$-PnPrOzi hydrogel $(n=0.11 ; K=132.8)$ [19]. Due to the additive, PMeOx- $b$-PnPrOzi/clay hydrogel $(20 \mathrm{wt} \% / 1.6 \mathrm{wt} \%)$ exhibited a higher viscosity than pristine $\mathrm{PMeOx}-b$-PnPrOzi hydrogel across the entire investigated shear rate range. The viscosity of $\mathrm{PMeOx}-b$-PnPrOzi/clay hydrogel decreases over five orders of magnitude as the shear rate increases from 0.01 to $1000 \mathrm{~s}^{-1}$. Such strong shear thinning is a highly desirable feature for materials used for (bio)printing. The highly shearthinning behavior enables efficient flow of the biomaterial ink through fine nozzles, while the rapid recovery to high viscosity leads to high shape fidelity. In addition, the hysteresis loop tests were also performed by subjecting the pristine and hybrid hydrogel to a loop of increasing shear and subsequent decreasing shear rate (Figure S5a, b, Supporting Information). Surprisingly, both samples did not show a thixotropic behavior, but were rather rheopectic. While the hysteresis in absolute numbers was more pronounced for the hybrid hydrogel 
Figure 5 a Viscosity depending on the applied shear rate for hydrogels at $37{ }^{\circ} \mathrm{C}$ including power-law fit. b Steady stress sweeps for hydrogels at $37{ }^{\circ} \mathrm{C}$. The intersection of the dashed lines is commonly referred to as the yield stress (arrows).

c Changes of viscosity with time at alternant shear rates of $0.1 \mathrm{~s}^{-1}$ and $100 \mathrm{~s}^{-1}$ at $37{ }^{\circ} \mathrm{C}$. d A more detailed view of c for better visibility of the recovery property.

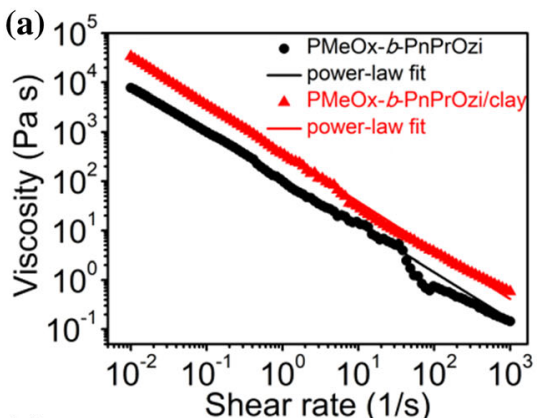

(c)

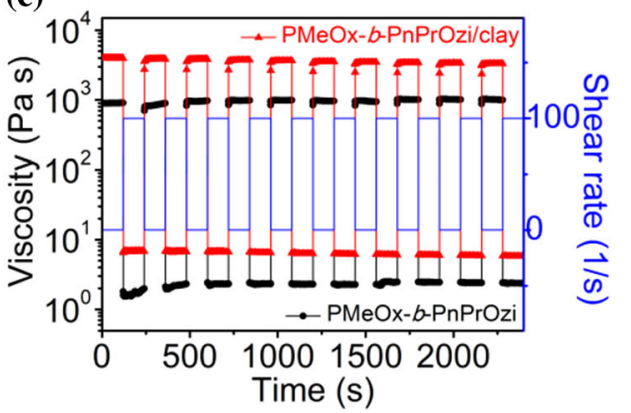

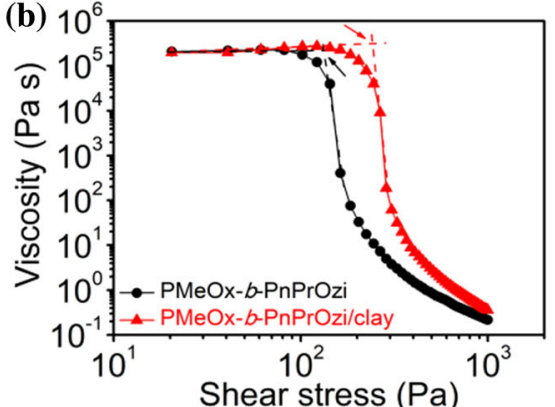

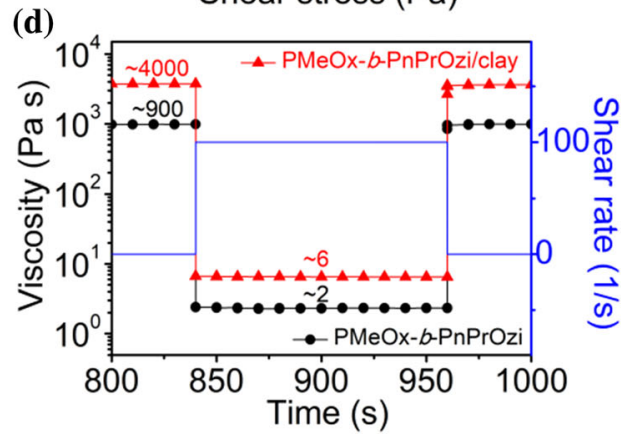

(Figure S5a) as the shear stress was much higher for the hybrid hydrogel, the relative hysteresis was the same for both samples, as can be seen readily after data normalization (Figure S5b).

The yield stress is considered the minimum stress needed for a material to flow and is a very critical parameter preventing collapse of structures just being printed and therefore to achieve high shape fidelity [19]. Steady stress sweeps revealed that the clay addition enhanced the hybrid hydrogel yield stress to around $240 \mathrm{~Pa}$, compared to only $140 \mathrm{~Pa}$ for pristine PMeOx-b-PnPrOzi hydrogel (Fig. 5b). Finally, the ability of the materials to recover to their original viscosity after extrusion was examined through the rotational recovery rheological measurements. Corroborating previous results (Fig. 4c), excellent viscosity recovery within seconds was observed and $\sim 85 \%$ recovery was observed even after ten consecutive cycles (Fig. 5c). At low shear rates $\left(0.1 \mathrm{~s}^{-1}\right)$, the $\mathrm{PMeOx}-b$-PnPrOzi/clay hybrid hydrogel shows a 4.4 times higher quasi-static viscosity $(\approx 4000 \mathrm{~Pa} \mathrm{~s})$ compared to the pristine PMeOx- $b$-PnPrOzi hydrogel ( $\approx 900 \mathrm{~Pa}$ s). The profound viscosity change of around 2.5 order of magnitude within seconds under the alternant of low $\left(0.1 \mathrm{~s}^{-1}\right)$ and high $\left(100 \mathrm{~s}^{-1}\right)$ shear rates is observed for both samples, i.e., with and without clay (Fig. $5 \mathrm{~d}$ ). This will likely contribute to high-resolution and high structure fidelity during extrusion-based 3D printing.

\section{Printability of PMeOx- $b$-PnPrOzi/clay} hydrogels

After extensive rheological analysis, the printability of the potential biomaterial ink was assessed using extrusion-based printing technology (Figure S5c, Video S3, Supporting Information). For printing, the hybrid biomaterial ink was kept at room temperature and printed onto a stage also at room temperature. However, for printing preparation, the thermogelling property is highly beneficial, as it facilitates mixing of the material and filling of the syringe in the cold, liquid state. Initially, 2D patterns with stepwise increasing strand distances varying from 0.75 to $1,1.25$ and $1.5 \mathrm{~mm}$ were printed using an extrusion pressure of $110 \mathrm{kPa}$ for assessment of strand fusion and the minimal strand-tostrand distance. Addition of the clay shows a clear benefit. When the pristine PMeOx-b-PnPrOzi was printed, strand fusion was observed at strand-to-strand distances below $1.25 \mathrm{~mm}$, marking this as the minimal strand-to-strand distance (red arrows, Fig. 6a). In addition, for a $5 \times 5$ grid pattern printed using pristine PMeOx-b-PnPrOzi, a low-quality printing was observed as indicated by red circles in Fig. 6b, again to fiber fusion at intersections. In contrast, with the hybrid hydrogel ink, the same grid was printed without any notable strand fusion or structural collapse. The printed strands remain excellently defined. This can be attributed to the improved rheological properties. Improved $G^{\prime}$ and yield stress in combination with an increased 
(a)
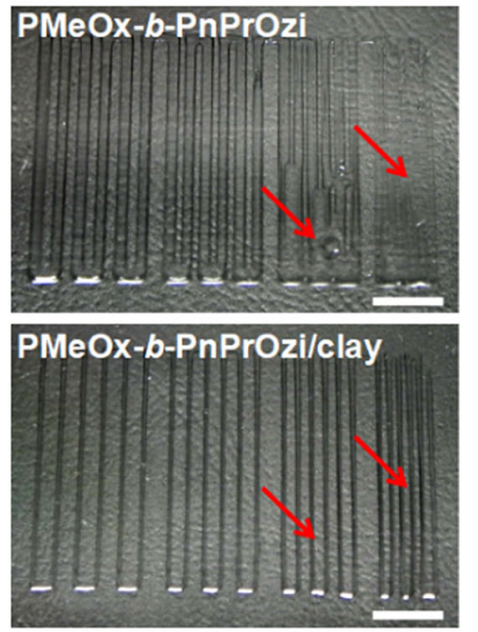

(b)

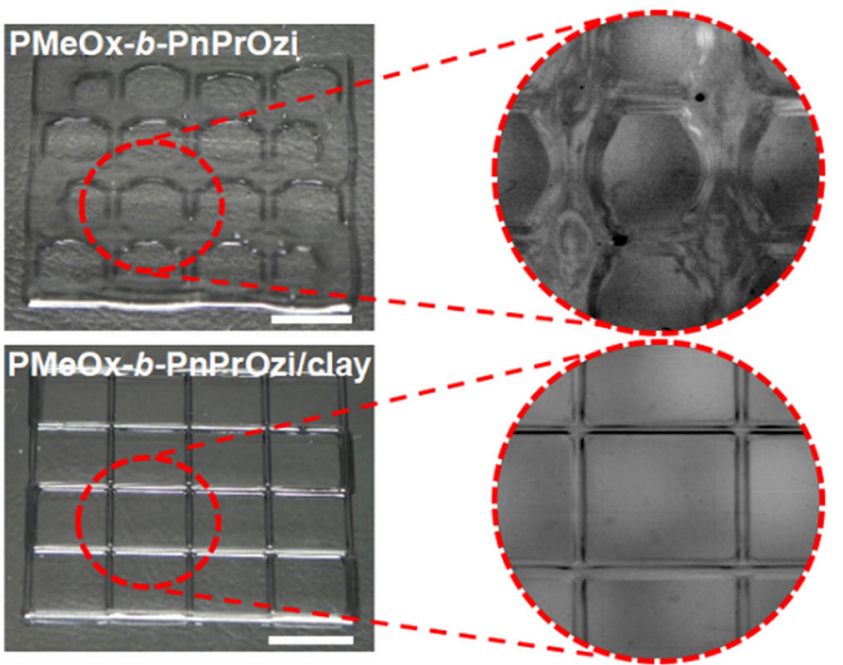

(c)
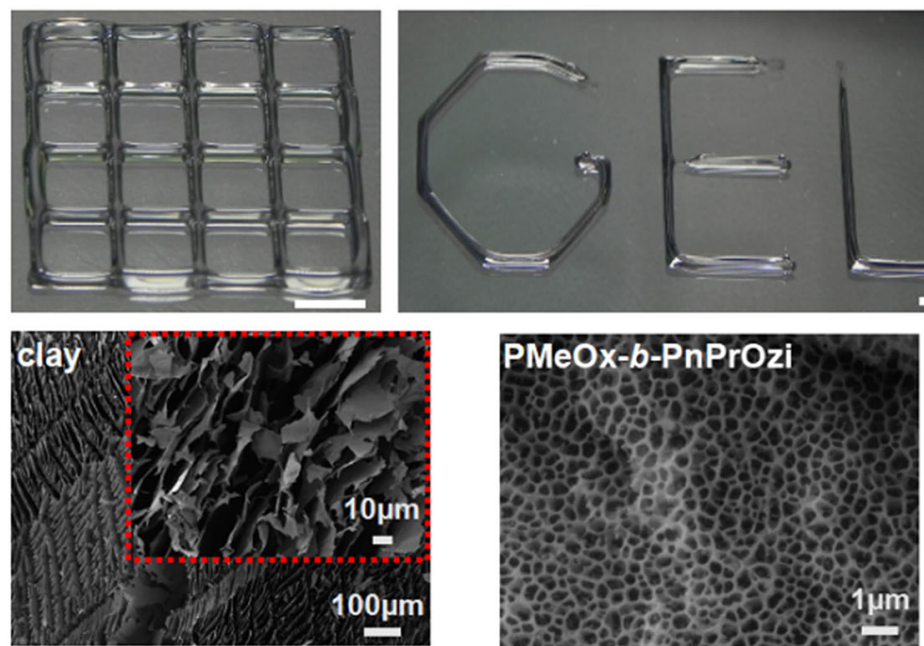
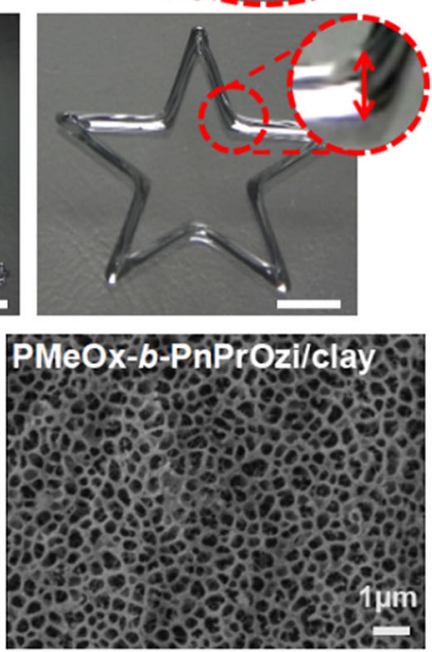

(d)

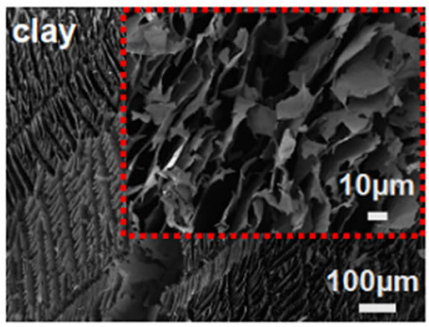

Figure 6 a 2D printed patterns for assessing the minimal strand-tostrand distance and shape fidelity of biomaterial inks (red arrows are guiding for eyes). $\mathbf{b}$ Optical and stereomicroscopic images of the printed constructs composed with three layers of $5 \times 5$ orthogonal strands with a base area of $20 \times 20 \mathrm{~mm}^{2}$ (amplified in dashed red

shear-thinning resulting in this highly significant improvement in the shape fidelity after printing. As mentioned previously, Gao and Gillispie suggested tan $\delta$ values in the range of $0.25-0.45$ as ideal for printing when using gelatin-alginate composite bioink [60]. With $\tan \delta \approx 0.05$ for the present hybrid material, we are clearly outside of this suggested range and therefore conclude that $\tan \delta$ alone is not well suited to assess printability across different materials. In contrast, our results suggest that different types of materials may require different parameters. Therefore, the search for a universally predictive parameter for good printability is still ongoing. circles). c Photographic images of 3D printed six layers of a $5 \times 5$ woodpile structure, letters "GEL" and a five-pointed star with PMeOx- $b$-PnPrOzi/clay hydrogel. d SEM images of clay and cryoSEM images of PMeOx- $b$-PnPrOzi and PMeOx- $b$-PnPrOzi/clay. Scale bars in $\mathbf{a}-\mathbf{c}$ represent $5 \mathrm{~mm}$.

Furthermore, the improved printing versatility of the PMeOx- $b$-PnPrOzi/clay hydrogel is supported by printing various shapes with six stacked layers [e.g., $5 \times 5$ woodpile (extrusion pressure of $220 \mathrm{kPa}$ ), letters "GEL" and a five-pointed star (extrusion pressure of $190 \mathrm{kPa})]$. The improvement in printability is also obvious when inspecting and comparing the smooth and uniform strands of the hybrid biomaterial ink with the pristine prints. Moreover, these printing experiments clearly demonstrate the self-supporting capability of the hybrid hydrogel, as opposed to the pristine block copolymer hydrogel (Fig. 6a-c; Figure S6, Supporting Information). The grid structure, intersections 
and sharp corners of the star are excellently resolved. It is well known that aqueous clay dispersions form a house-of-cards microstructure. From rheological measurements, it became apparent that the addition of clay strengthens the overall structure of the hybrid hydrogel as evidenced by an increase in $G^{\prime}$. However, how the microstructure is affected is unclear. Therefore, we investigated the morphology of the clay using SEM and of the block copolymer hydrogel and the block copolymers/clay hybrid hydrogel using cryoSEM (Fig. 6d) after flash freezing in liquid nitrogen slush, respectively. Clearly, the house-of-cards structure of the aqueous clay dispersion could be confirmed. It could be hypothesized that this structure could function as a self-supporting internal scaffold contributing to the improvement in viscoelastic properties and printing performance in the $\mathrm{PMeOx}-b$ PnPrOzi/clay hydrogels. However, there is no obvious difference observable between the two hydrogel structures. Both the PMeOx- $b$-PnPrOzi and PMeOx- $b$ PnPrOzi/clay hydrogels show a typical hydrogel microstructure with a relatively uniform, macroporous structure. Interestingly, the addition of clay does not seem to have a marked effect on the pore size.

\section{Conclusions}

In summary, we have successfully adjusted the rheological properties of the synthetic thermoresponsive hydrogel based on PMeOx- $b$-PnPrOzi by the addition of clay Laponite XLG. The thermoresponsive character was retained with an only slightly decreased gelation temperature. More importantly, the PMeOx$b$-PnPrOzi/clay hybrid hydrogels exhibited markedly enhanced viscoelastic properties such as storage modulus, yield stress and increased shear thinning. Such changes are highly desirable for extrusionbased 3D (bio)printing and resulted in a significantly improved printability. Printed strand fusion was markedly suppressed, which allowed drastically improved stacking and shape fidelity. This newly developed thermoresponsive hydrogel is very promising to expand the options available to researchers for 3D bioprinting. Furthermore, the new hybrid material platform could be interesting for many other research fields, such as controlled drug delivery and release, versatile support bath material for fluid extrusion printing, sacrificial material templates in fabrication of microfluidic devices and thermoresponsive self-protection.

\section{Supporting information}

Preparation of PMeOx- $b$-PnPrOzi/clay hybrid hydrogels. Detailed description of ${ }^{1} \mathrm{H}-\mathrm{NMR}$ of block copolymer PMeOx-b-PnPrOzi; XRD analysis; transmittance tests in the visible range; TGA and DSC analysis; Loss factor $\tan \delta$ of hydrogels; Hysteresis loop tests of the hydrogels; Extrusion-based printing equipment; Photographs of the 3D printed constructs (Figures S1-S6, and Table S1, S2) (PDF). Supporting videos: Sol-gel transition of $\mathrm{PMeOx}-b-\mathrm{PnPrOzi} /$ clay hydrogel (AVI); Injection of PMeOx-b-PnPrOzi/clay hydrogel (AVI); Printing of PMeOx-b-PnPrOzi/clay hydrogel (AVI).

\section{Acknowledgements}

This work was supported by the China Scholarship Council (CSC) and University Würzburg. The authors would like to gratefully acknowledge support by the Deutsche Forschungsgemeinschaft (DFG, German Research Foundation)-Project Number 326998133-TRR 225 (Subproject A03), awarded to R.L. Furthermore, we thank the Deutsche Forschungsgemeinschaft for funding the crossbeam scanning electron microscope Zeiss CB 340 (INST 105022/58-1 FUGG) within the DFG State Major Instrumentation Programme.

\section{Funding}

Open Access funding provided by Projekt DEAL.

\section{Compliance with ethical standards}

Conflict of interest RL is listed as inventor on a patent pertinent to some materials in the present work.

Electronic supplementary material: The online version of this article (https://doi.org/10.1007/s108 53-020-05190-5) contains supplementary material, which is available to authorized users. 
Open Access This article is licensed under a Creative Commons Attribution 4.0 International License, which permits use, sharing, adaptation, distribution and reproduction in any medium or format, as long as you give appropriate credit to the original author(s) and the source, provide a link to the Creative Commons licence, and indicate if changes were made. The images or other third party material in this article are included in the article's Creative Commons licence, unless indicated otherwise in a credit line to the material. If material is not included in the article's Creative Commons licence and your intended use is not permitted by statutory regulation or exceeds the permitted use, you will need to obtain permission directly from the copyright holder. To view a copy of this licence, visit http://creativecommons.org/licen ses/by $/ 4.0 /$.

Electronic supplementary material: The online version of this article (https://doi.org/10.1007/s108 53-020-05190-5) contains supplementary material, which is available to authorized users.

\section{References}

[1] Lee W, Debasitis JC, Lee VK, Lee JH, Fischer K, Edminster K, Park JK, Yoo SS (2009) Multi-layered culture of human skin fibroblasts and keratinocytes through three-dimensional freeform fabrication. Biomaterials 30:1587-1595

[2] Cohen DL, Lo W, Tsavaris A, Peng D, Lipson H, Bonassar LJ (2011) Increased mixing improves hydrogel homogeneity and quality of three-dimensional printed constructs. Tissue Eng Part C Methods 17:239-248

[3] Rhee S, Puetzer JL, Mason BN, Reinhart-King CA, Bonassar LJ (2016) 3D bioprinting of spatially heterogeneous collagen constructs for cartilage tissue engineering. ACS Biomater Sci Eng 2:1800-1805

[4] Cui XF, Breitenkamp K, Finn MG, Lotz M, D'Lima DD (2012) Direct human cartilage repair using three-dimensional bioprinting technology. Tissue Eng Part A 18:1304-1312

[5] Abbadessa A, Mouser VHM, Blokzijl MM, Gawlitta D, Dhert WJA, Hennink WE, Malda J, Vermonden T (2016) A synthetic thermosensitive hydrogel for cartilage bioprinting and its biofunctionalization with polysaccharides. Biomacromolecules 17:2137-2147

[6] You F, Eames BF, Chen X (2017) Application of extrusionbased hydrogel bioprinting for cartilage tissue engineering. Int J Mol Sci 18:1597
[7] Tang D, Tare RS, Yang LY, Williams DF, Ou KL, Oreffo ROC (2016) Biofabrication of bone tissue: approaches, challenges and translation for bone regeneration. Biomaterials $83: 363-382$

[8] Ozbolat IT (2015) Bioprinting scale-up tissue and organ constructs for transplantation. Trends Biotechnol 33:395-400

[9] Wu W, DeConinck A, Lewis JA (2011) Omnidirectional printing of $3 \mathrm{D}$ microvascular networks. Adv Mater 23:H178-H183

[10] Malda J, Visser J, Melchels FP, Jungst T, Hennink WE, Dhert WJA, Groll J, Hutmacher DW (2013) 25th anniversary article: engineering hydrogels for biofabrication. Adv Mater 25:5011-5028

[11] Groll J, Boland T, Blunk T, Burdick JA, Cho DW, Dalton PD, Derby B, Forgacs G, Li Q, Mironov VA, Moroni L, Nakamura M, Shu WM, Takeuchi S, Vozzi G, Woodfield TBF, Xu T, Yoo JJ, Malda J (2016) Biofabrication: reappraising the definition of an evolving field. Biofabrication 8:013001

[12] Pedde RD, Mirani B, Navaei A, Styan T, Wong S, Mehrali M, Thakur A, Mohtaram NK, Bayati A, Dolatshahi-Pirouz A, Nikkhah M, Willerth SM, Akbari M (2017) Emerging biofabrication strategies for engineering complex tissue constructs. Adv Mater 29:1606061

[13] Levato R, Visser J, Planell JA, Engel E, Malda J, MateosTimoneda MA (2014) Biofabrication of tissue constructs by $3 \mathrm{D}$ bioprinting of cell-laden microcarriers. Biofabrication 6:035020

[14] Ozbolat IT, Hospodiuk M (2016) Current advances and future perspectives in extrusion-based bioprinting. Biomaterials 76:321-343

[15] Murphy SV, Atala A (2014) 3D bioprinting of tissues and organs. Nat Biotechnol 32:773-785

[16] Placone JK, Engler AJ (2018) Recent advances in extrusionbased 3D printing for biomedical applications. Adv Healthc Mater 7:1701161

[17] Groll J, Burdick J, Cho D, Derby B, Gelinsky M, Heilshorn S, Jüngst T, Malda J, Mironov V, Nakayama K (2018) A definition of bioinks and their distinction from biomaterial inks. Biofabrication 11:013001

[18] Jüngst T, Smolan W, Schacht K, Scheibel T, Groll J (2016) Strategies and molecular design criteria for 3D printable hydrogels. Chem Rev 116:1496-1539

[19] Paxton N, Smolan W, Bock T, Melchels F, Groll J, Jungst T (2017) Proposal to assess printability of bioinks for extrusion-based bioprinting and evaluation of rheological properties governing bioprintability. Biofabrication 9:044107

[20] Jia J, Richards DJ, Pollard S, Tan Y, Rodriguez J, Visconti RP, Trusk TC, Yost MJ, Yao H, Markwald RR, Mei Y (2014) 
Engineering alginate as bioink for bioprinting. Acta Biomater 10:4323-4331

[21] Cidonio G, Alcala-Orozco CR, Lim KS, Glinka M, Mutreja I, Kim YH, Dawson JI, Woodfield TBF, Oreffo ROC (2019) Osteogenic and angiogenic tissue formation in high fidelity nanocomposite laponite-gelatin bioinks. Biofabrication 11:035027

[22] Ouyang LL, Highley C, Rodell C, Sun W, Burdick J (2016) $3 \mathrm{D}$ printing of shear-thinning hyaluronic acid hydrogels with secondary crosslinking. ACS Biomater Sci Eng 2:1743-1751

[23] Hockaday LA, Kang KH, Colangelo NW, Cheung PYC, Duan B, Malone E, Wu J, Girardi LN, Bonassar LJ, Lipson H, Chu CC, Butcher JT (2012) Rapid 3D printing of anatomically accurate and mechanically heterogeneous aortic valve hydrogel scaffolds. Biofabrication 4:035005

[24] Xu CC, Lee WH, Dai GH, Hong Y (2018) Highly elastic biodegradable single-network hydrogel for cell printing. ACS Appl Mater Interfaces 10:9969-9979

[25] Wu CJ, Schmidt G (2009) Thermosensitive and dissolution properties in nanocomposite polymer hydrogels. Macromol Rapid Commun 30:1492-1497

[26] Sun KS, Raghavan SR (2010) Thermogelling aqueous fluids containing low concentrations of pluronic F127 and laponite nanoparticles. Langmuir 26:8015-8020

[27] Chang CW, van Spreeuwel A, Zhang C, Varghese S (2010) PEG/clay nanocomposite hydrogel: a mechanically robust tissue engineering scaffold. Soft Matter 6:5157-5164

[28] Luo YX, Zhai D, Huan ZG, Zhu HB, Xia LG, Chang J, Wu CT (2015) Three-dimensional printing of hollow-strutspacked bioceramic scaffolds for bone regeneration. ACS Appl Mater Interfaces 7:24377-24383

[29] Trachsel L, Broguiere N, Rosenboom JG, Zenobi-Wong M, Benetti EM (2018) Enzymatically crosslinked poly(2-alkyl2-oxazoline) networks for $3 \mathrm{D}$ cell culture. J Mater Chem B 6:7568-7572

[30] Dargaville TR, Park JR, Hoogenboom R (2018) Poly(2-oxazoline) hydrogels: state-of-the-art and emerging applications. Macromol Biosci 18:1800070

[31] Luxenhofer R, Han Y, Schulz A, Tong J, He Z, Kabanov AV, Jordan R (2012) Poly(2-oxazoline)s as polymer therapeutics. Macromol Rapid Commun 33:1613-1631

[32] Lorson T, Lübtow MM, Wegener E, Haider MS, Borova S, Nahm D, Jordan R, Sokolski-Papkov M, Kabanov AV, Luxenhofer R (2018) Poly(2-oxazoline)s based biomaterials: a comprehensive and critical update. Biomaterials 178:204-280

[33] Hartlieb M, Kempe K, Schubert US (2015) Covalently cross-linked poly(2-oxazoline) materials for biomedical applications-from hydrogels to self-assembled and templated structures. J Mater Chem B 3:526-538

[34] Jerca FA, Anghelache AM, Ghibu E, Cecoltan S, Stancu IC, Trusca R, Vasile E, Teodorescu M, Vuluga DM, Hoogenboom R, Jerca VV (2018) Poly(2-isopropenyl-2-oxazoline) hydrogels for biomedical applications. Chem Mater 30:7938-7949

[35] Zahoranova A, Kronekova Z, Zahoran M, Chorvat D, Janigova I, Kronek J (2016) Poly(2-oxazoline) hydrogels crosslinked with aliphatic bis(2-oxazoline)s: properties, cytotoxicity, and cell cultivation. J Polym Sci Part A Polym Chem 54:1548-1559

[36] Dargaville TR, Forster R, Farrugia BL, Kempe K, Voorhaar L, Schubert US, Hoogenboom R (2012) Poly(2-oxazoline) hydrogel monoliths via thiol-ene coupling. Macromol Rapid Commun 33:1695-1700

[37] Lorson T, Jaksch S, Lübtow MM, Jüngst T, Groll J, Lühmann T, Luxenhofer R (2017) A thermogelling supramolecular hydrogel with sponge-Like morphology as a cytocompatible bioink. Biomacromolecules 18:2161-2171

[38] Lübtow MM, Mrlik M, Hahn L, Altmann A, Beudert M, Lühmann T, Luxenhofer R (2019) Temperature-dependent rheological and viscoelastic investigation of a poly(2methyl-2-oxazoline)-b-poly(2-iso-butyl-2-oxazoline)-bpoly(2-methyl-2-oxazoline)-based thermogelling hydrogel. J Funct Biomater 10:36

[39] Monnery BD, Hoogenboom R (2019) Thermoresponsive hydrogels formed by poly(2-oxazoline) triblock copolymers. Polym Chem 10:3480-3487

[40] Chimene D, Kaunas R, Gaharwar AK (2020) Hydrogel bioink reinforcement for additive manufacturing: a focused review of emerging strategies. Adv Mater 32:1902026

[41] Parak A, Pradeep P, du Toit LC, Kumar P, Choonara YE, Pillay V (2019) Functionalizing bioinks for 3D bioprinting applications. Drug Discov Today 24:198-205

[42] Gungor-Ozkerim PS, Inci I, Zhang YS, Khademhosseini A, Dokmeci MR (2018) Bioinks for 3D bioprinting: an overview. Biomater Sci 6:915-946

[43] Hong SM, Sycks D, Chan HF, Lin ST, Lopez GP, Guilak F, Leong KW, Zhao XH (2015) 3D printing of highly stretchable and tough hydrogels into complex, cellularized structures. Adv Mater 27:4035-4040

[44] Mignon A, Pezzoli D, Prouve E, Levesque L, Arslan A, Pien N, Schaubroeck D, Van Hoorick J, Mantovani D, Van Vlierberghe S, Dubruel P (2019) Combined effect of Laponite and polymer molecular weight on the cell-interactive properties of synthetic PEO-based hydrogels. React Funct Polym 136:95-106

[45] Chimene D, Peak CW, Gentry JL, Carrow JK, Cross LM, Mondragon E, Cardoso GB, Kaunas R, Gaharwar AK (2018) 
Nanoengineered ionic-covalent entanglement (NICE) bioinks for 3D bioprinting. ACS Appl Mater Interfaces 10:9957-9968

[46] Jin YF, Shen YY, Yin J, Qian J, Huang Y (2018) Nanoclaybased self-supporting responsive nanocomposite hydrogels for printing applications. ACS Appl Mater Interfaces 10:10461-10470

[47] Murugesan S, Scheibel T (2020) Copolymer/clay nanocomposites for biomedical applications. Adv Funct Mater 30:1908101

[48] Gao Q, Niu X (2019) 3D printing of complex GelMA-based scaffolds with nanoclay. Biofabrication 11:035006

[49] Ahlfeld T, Cidonio G, Kilian D, Duin S, Akkineni A, Dawson J, Yang S, Lode A, Oreffo R, Gelinsky M (2017) Development of a clay based bioink for 3D cell printing for skeletal application. Biofabrication 9:034103

[50] Jin Y, Liu C, Chai W, Compaan A, Huang Y (2017) Selfsupporting nanoclay as internal scaffold material for direct printing of soft hydrogel composite structures in air. ACS Appl Mater Interfaces 9:17457-17466

[51] Wang CH, Hwang YS, Chiang PR, Shen CR, Hong WH, Hsiue GH (2012) Extended release of bevacizumab by thermosensitive biodegradable and biocompatible hydrogel. Biomacromolecules 13:40-48

[52] Compaan AM, Song K, Huang Y (2019) Gellan fluid gel as a versatile support bath material for fluid extrusion bioprinting. ACS Appl Mater Interfaces 11:5714-5726

[53] Ozbolat V, Dey M, Ayan B, Ozbolat IT (2019) Extrusionbased printing of sacrificial carbopol ink for fabrication of microfluidic devices. Biofabrication 11:034101
[54] Shi Y, Ha H, Al-Sudani A, Ellison CJ, Yu GH (2016) Thermoplastic elastomer-enabled smart electrolyte for thermoresponsive self-protection of electrochemical energy storage devices. Adv Mater 28:7921-7928

[55] Sinnwell S, Ritter H (2006) Microwave accelerated polymerization of 2-phenyl-5,6-dihydro-4H-1,3-oxazine: kinetics and influence of end-groups on glass transition temperature. Macromol Rapid Commun 27:1335-1340

[56] Luxenhofer R, Schulz A, Roques C, Li S, Bronich TK, Batrakova EV, Jordan R, Kabanov AV (2010) Doubly amphiphilic poly(2-oxazoline)s as high-capacity delivery systems for hydrophobic drugs. Biomaterials 31:4972-4979

[57] Lorson T (2019) Novel poly(2-oxazoline) based bioinks. $\mathrm{Ph} . \mathrm{D}$. thesis Julius-Maximilians-Universität Würzburg

[58] Le Coeur C, Lorthioir C, Feoktystov A, Wu B, Volet G, Amiel C (2021) Laponite/poly(2-methyl-2-oxazoline) hydrogels: interplay between local structure and rheological behaviour. J Coll Interface Sci 582:149-158

[59] Boucenna I, Royon L, Colinart P (2009) Effect of laponite clay particles on thermal and rheological properties of pluronic triblock copolymer. J Therm Anal Calorim 98:119-123

[60] Gao T, Gillispie GJ, Copus JS, PR AK, Seol Y-J, Atala A, Yoo JJ, Lee SJ, (2018) Optimization of gelatin-alginate composite bioink printability using rheological parameters: a systematic approach. Biofabrication 10:034106

Publisher's Note Springer Nature remains neutral with regard to jurisdictional claims in published maps and institutional affiliations. 